\title{
ISI: Recent Technology and Science
}

\author{
David D. Snyder Hale, J. Weiner, and C. H. Townes \\ The ISI Array \\ Space Sciences Laboratory \\ University of California at Berkeley \\ Berkeley, CA, 94720-7450, USA
}

\begin{abstract}
When we last reported the status of the U.C. Berkeley Infrared Spatial Interferometer (ISI) in $2002,{ }^{1}$ we presented simulations, based upon our two-telescope experience, for the expected performance of a three-telescope array that would be capable of measuring three simultaneous visibilities and one closure phase at mid-infrared wavelengths.

The ISI is now fully operational as an imaging array and is routinely making fringe visibility and closure phase measurements of late-type stars in the $9-12 \mu \mathrm{m}$ wavelength region. We describe here the technology which is currently in use, along with actual measurements and preliminary $11.15 \mu \mathrm{m}$ (one-dimensional) image reconstructions.
\end{abstract}

Keywords: instrumentation: closure phase: infrared: interferometry:

\section{INTRODUCTION}

The U.C. Berkeley ISI Array consists of three movable, $1.65 \mathrm{~m}$ aperture Pfund-type telescopes, operating as an interferometer in the mid-IR $(9-12 \mu \mathrm{m})$ wavelength region. The Pfund design is one which is inherently low to the ground and hence quite stable against vibrations, making it well-suited for use as an interferometer element. The interferometer array is situated at the Mount Wilson Observatory, where our movable telescopes can be somewhat freely positioned to form baselines ranging from $4 \mathrm{~m}$ to $70 \mathrm{~m}$ in a variety of different orientations.

When we last presented the status of the ISI at the SPIE Astronomical Telescopes \& Instrumentation Symposium in $2002,{ }^{1}$ only the original two ISI telescopes (which have been in continual use since 1988) were operational. At that time we analyzed the potential performance expected from a 3-telescope array, from a signal-to-noise perspective, using simulated data based upon our extensive experience with the 2-telescope interferometer system. Since that time we have succeeded in making all three telescopes operational as an interferometric array, implementing three-way fringe production, and formulating a technique for determining closure phase. On 2003 Jul 09, the ISI Array recorded three simultaneous fringe visibilities and one closure phase on a stellar source, marking the first such measurements ever made in the mid-IR wavelength region. Observations have since continued on many astronomical sources using a co-linear array of East-West baselines $(4 \mathrm{~m}, 8 \mathrm{~m}, 12 \mathrm{~m})$.

We have organized this paper to begin with a brief discussion of the scientific motivation behind the expansion of the capabilities of the ISI to include triple fringe and closure phase measurements. Because we have already described many of the fundamental properties of the ISI in a few recent papers, ${ }^{1,2}$ we will then follow with only a short summary of the overall ISI system, allowing for slight changes, made where appropriate, to reflect the current status. Finally, the balance and emphasis of this paper will be devoted to the recent technological aspects which have been pivotal in providing three simultaneous fringes and closure phase measurements at mid-IR wavelengths. This will include a discussion of the interferometer performance and a sample of some actual results which have been obtained with the new system.

Further author information: (Send correspondence to D.D.S.H.)

D.D.S.H.: E-mail: david@isi.mtwilson.edu, Address: Mount Wilson Observatory, Mount Wilson, CA, 91023, USA 


\section{SCIENTIFIC MOTIVATION}

Since its inception in the late 1980s, the primary emphasis of the observation program of the ISI has been focussed on evolved stars and their dusty environments. Not only is the thermal infrared region particularly important for being able to detect the relatively cooler material surrounding stars, but it also allows one to observe the star through the dust shell, with minimal limb darkening effect. Stars typically appear much larger at this wavelength than at other wavelengths, and additional work, both in terms of precision measurements and good models, will be required to better explain this.

The commissioning of new, longer $(\approx 50 \mathrm{~m})$ baselines in 1999 allowed the ISI to begin resolving stellar disks and measuring diameters through 2001 as recently reported by Weiner et al. ${ }^{3-5}$ However, atmospheric density variations and uncertainties in precise pathlengths within the telescopes mean that useful fringe phase information with only two telescopes is not obtainable. Therefore, it had not been possible before now to obtain accurate high resolution information regarding source symmetry in the mid-IR, and reflection symmetric (usually spherical) models had to be used, despite some evidence that this was not correct. In the infrared especially, where dust formation is observed, it is quite possible that clumps or other asymmetries may exist.

With the addition of a third interferometer element, the ISI becomes capable of recovering much of the lost phase information distorted by path length uncertainties. Furthermore, one additional telescope triples the number of simultaneous baselines measured, allowing for a greatly expanded spatial frequency coverage without having to move telescopes. The upgraded ISI Array not only provides added source geometry information, but also enables us to obtain a range of baselines (which had previously required about a year because of the need to move telescopes) in just a single night of observation, with higher quality.

\section{SYSTEM OVERVIEW}

The fundamental characteristic which sets apart the ISI from other optical interferometers is its usage of heterodyne detection. Each telescope contains an independent heterodyne receiver ${ }^{6}$ which detects mid-IR starlight by combining, or mixing the stellar signal collected by the telescope with a fixed-frequency signal (generated by a tunable $\mathrm{CO}_{2}$ laser similar to the Freed ${ }^{7}$ design) onto a single-pixel nonlinear detector; the frequency of the laser is chosen to lie at the center of the band to be detected in the stellar source. The multiplication of these two signals produces a detector response which contains frequencies equal to the difference of the two input frequencies. These intermediate frequencies (IF) extend from approximately 200 to $2800 \mathrm{MHz}$, providing a double sideband (DSB) bandwidth of about $5 \frac{1}{2} \mathrm{GHz}$. Thus we down-convert a mid-IR signal into a radio signal. Only the frequency is changed; all of the amplitude and phase information contained in the original stellar signal is preserved in the IF signal.

Since each telescope's heterodyne receiver possesses its own laser, each of these lasers must remain in phase with each other in order that we might meaningfully correlate the IF signals to produce interference fringes. This is accomplished by using a fourth, external laser as a "master oscillator", to which all of the telescope lasers are phase-locked. Multiple feedback loops maintain the phase and frequency of each laser, and monitor and control the pathlength between each telescope. This master oscillator is a new addition to the ISI system in order to allow three-telescope operation; in the original two-telescope system, one telescope's laser was transferred to the other for use as a phase lock reference.

After heterodyne detection of the mid-IR stellar signal, the remaining signal handling required for interferometry is performed at these intermediate, radio frequencies (RF). The heterodyned stellar signals from each telescope are carried along coaxial cables to a central station. There, variable lengths of cables are inserted or removed under computer control as necessary to account for the changing geometrical delay of a stellar wavefront arriving at the various interferometer elements, caused by diurnal motion. Because of the additional cable lengths required by the three-telescope array, temperature sensors are placed at several locations in contact with the cables, in order to determine thermal expansion effects. Inside the central control facility, in a manner analogous to superimposing optical beams on a detector (as is done by most other interferometers), our beam combination is accomplished first in a pairwise fashion by multiplying the pairs of appropriately delayed RF signals in an electronic correlator. Local laser oscillators in the three telescopes are locked in phase, but at slightly different frequencies. Hence the resultant fringe signals produced from each of the three baselines are 
modulated at unique audio frequencies $\left(\mathrm{AF}, \nu_{F}<200 \mathrm{~Hz}\right)$. The AF-modulated fringes are then sampled with an analog-to-digital converter (ADC) controlled by a real-time VME computer and recorded on disk for later analysis. Additional information, such as uncorrelated infrared power recieved by each individual telescope, along with dozens of other system parameters, are also recorded on disk. A suite of software tools written in IDL is used for reading, processing, and analyzing the recorded data following the methods outlined by Hale et al. ${ }^{1}$ The bispectrum is formed in software, as outlined in section 4.1.

A determination of fringe power on a given baseline is then a simple matter of examining the power spectrum recorded by the ADC, integrating around the frequency at which that baseline's fringes were modulated. Lessthan-ideal atmospheric seeing conditions cause phase fluctuations in the stellar signal, which are manifested as frequency fluctuations in the AF-modulated fringe signals. We can then recover much of the fringe power which would otherwise be lost simply by integrating over a larger bandwidth in the AF fringe power spectrum. Fringes recorded during typical seeing conditions on Mount Wilson ( $\sim 2$ to 3 arcseconds) are mostly contained within about $5 \mathrm{~Hz}$. Under unusually good seeing conditions, the fringes have had a frequency spread as small as $0.01 \mathrm{~Hz}$. Poor seeing ( $\sim 5$ to 6 arcseconds) may require a bandwidth of as much as $10 \mathrm{~Hz}$ to recover most of the fringe power. Observing in seeing worse than this requires still larger bandwidths in order to try to recover fringe power, which decreases the signal-to-noise ratio; hence it becomes inefficient to perform measurements during seeing conditions $\gtrsim 6$ arcseconds, which fortunately are rarely experienced on Mount Wilson.

\section{SYSTEM PERFORMANCE}

In discussing the system performance, it is useful to outline the method for obtaining the bispectrum measurement. As mentioned in section 3, fringes are recorded only pairwise, in three unique baseline pair combinations. The three interference fringes created by the possible pairs of telescopes are modulated at audio frequencies unique to each baseline. The criteria on the audio fringe frequencies are that they must sum to zero; each must be less than the Nyquist frequency; each must not be too close to $60 \mathrm{~Hz}$ or its harmonics; and each must not be too close to other pickup or harmonic frequencies present in the system, such as chopping frequencies, etc. A simplified form of a recorded fringe signal $F_{\overrightarrow{j k}}(t)$ between telescopes $j$ and $k$ can be written as

$$
F_{\overrightarrow{j k}}(t) \propto \sqrt{2 P_{j k}}\left[\frac{1}{2} e^{i\left(2 \pi \nu_{F_{j}} t+\phi \nu_{j k}+\varphi_{k}(t)-\varphi_{j}(t)\right)}+\frac{1}{2} e^{i\left(-2 \pi \nu_{F_{j}} t-\phi \nu_{j k}-\varphi_{k}(t)+\varphi_{j}(t)\right)}\right]
$$

where $P_{j k}$ represents the power in the fringe between interferometer elements $j$ and $k ; \nu_{F_{j}}$ is the (audio) fringe frequency for baseline $j$; $\phi \mathcal{\nu}_{j k}$ is the phase of the complex visibility between telescopes $j$ and $k$; and $\varphi_{j}(t)$ represents the fluctuation in pathlength to telescope $j$.

\subsection{Bispectrum}

The fringe signals of equation (1) are generated in hardware by multiplying the signals from a pair of telescopes together in an electronic correlator; the bispectrum is generated in software using these recorded signals from each of the three possible baselines. From Hale et al. ${ }^{1}$ (2003), we obtain

$$
\langle\text { bispectrum }\rangle=\frac{1}{N_{t_{c}}} \sum_{i} \operatorname{FFT}_{\nu_{12}}\left[F_{\overrightarrow{12}}\left(t_{i}+t_{c}\right) H\left(t_{c}\right)\right] \cdot \operatorname{FFT}_{\nu_{23}}\left[F_{2 \overrightarrow{3}}\left(t_{i}+t_{c}\right) H\left(t_{c}\right)\right] \cdot \operatorname{FFT}_{\nu_{31}}\left[F_{3 \overrightarrow{1}}\left(t_{i}+t_{c}\right) H\left(t_{c}\right)\right]
$$

where $\mathrm{FFT}_{\nu_{j k}}$ denotes the Fast Fourier Transform evaluated only at the single frequency component identified by the audio fringe frequency $\nu_{j k}$ between interferometer elements $j$ and $k$; the summation is done over the entire data set in steps of the coherence time $t_{c} ; N_{t_{c}}$ denotes the number of coherent bins of size $t_{c} ; H\left(t_{c}\right)$ denotes a Hanning window function of width equal to the coherence time, $t_{c}$. 


\subsection{Signal-to-Noise}

In order to obtain the most accurate fringe visibility measurement from the recorded signal of equation (1), one must use a wide enough bandwidth in audio-frequency space to ensure that all of the power in the AF-modulated fringe is encompassed. Using a large bandwidth can come at the cost of adding excess noise, particularly in poor atmospheric seeing conditions. This is because the fringe signal peaks at the fringe frequency and decreases over the bandwidth, whereas the noise power is uniform over the bandwidth. A measurement of the phase, however, does not require that all of the energy from the tails of a distribution be included for an accurate determination, and instead there can be a slight advantage to using a somewhat narrower bandwidth. These effects are illustrated in figure 1.
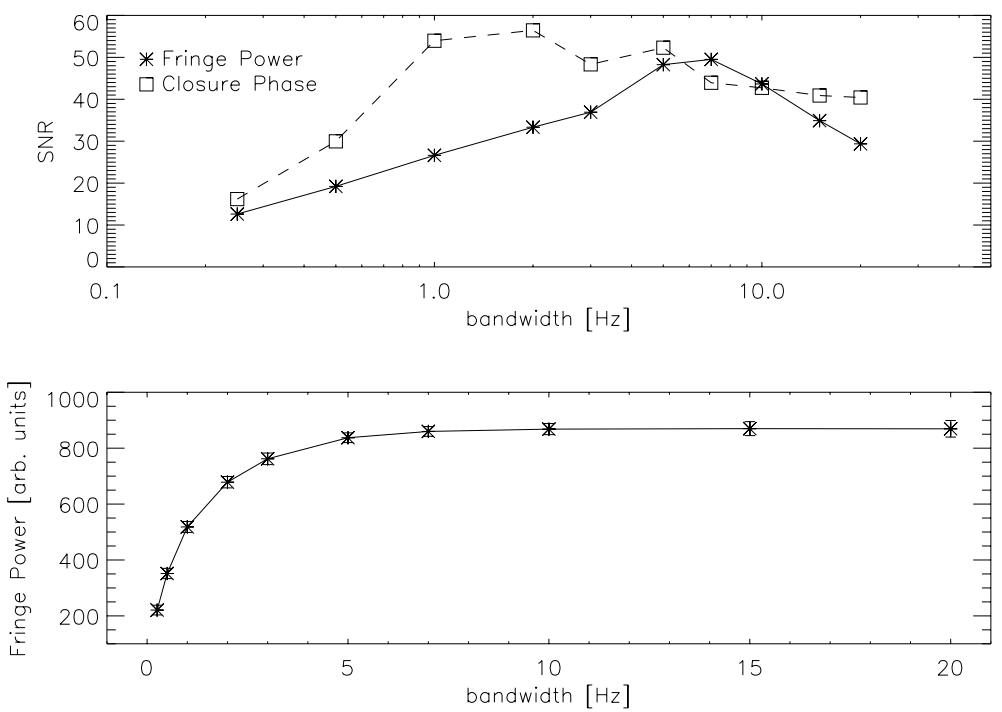

Figure 1. The effect of bandwidth on the signal-to-noise ratio (SNR). The top panel shows the SNR in both the closure phase and the fringe power for data recorded on $\alpha$ Tau on UT 2003 Oct 23, measured as a function of fringe bandwidth. The bottom panel shows the measured fringe power (for the same dataset) as a function of bandwidth. The error bars represent the standard deviation of the mean of 17 different 2 minute integrations recorded over a $\sim 90$ minute period.

An earlier theoretical discussion ${ }^{1}$ led to an expected precision in the closure phase of approximately $3.6^{\circ}$, and a series of simulations were in good agreement with this $\left(\sim 3^{\circ}\right)$. Our actual measurements are also in good agreement with this, and in some cases they are substantially better, on the order of a degree or less as shown in the following section.

\subsection{Closure Phase Results}

Fourteen stars have been observed at various positions above the new ISI Array since "first triple fringe" was achieved on $2003 \mathrm{Jul}$ 09; three visibilities and one closure have been measured for each of them. Stars which are believed to be point sources have been measured to have the same closure phase, while measurements of many other stars reveal different closure phases, in many cases changing as their position changes with respect to the array.

Two examples of well-known point-source calibrators at $11 \mu \mathrm{m}$ are the K-type Giants $\alpha$ Tauri and $\alpha$ Bootis, which are also good candidates for phase closure calibration. Results of closure phase measurements for $\alpha$ Tau and $\alpha$ Boo are shown in figure 2 and figure 3, respectively. Numerous measurements have been made on each of several different nights over the course of many months, and the data has been grouped according to each "epoch", or closely-spaced dates of observation, in order to be able to distinguish any possible instrumental variation. No such variation of significance is seen. 


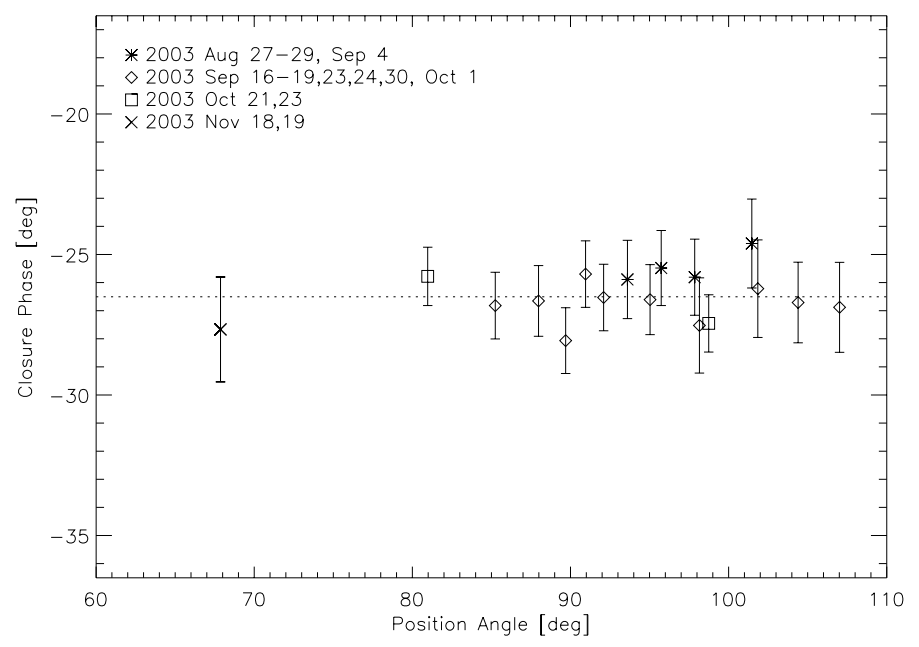

Figure 2. Closure phase data for our calibrator star $\alpha$ Tau, group-averaged over closely spaced dates observation as indicated in the legend above. Our instrumental calibration factor of $(-26.51 \pm 0.32)^{\circ}$, computed as the weighted average of all $\alpha$ Tau measurements, is shown here as a dotted line. The data span a range in spatial frequency $\sim(6-11) \times 10^{5} \mathrm{rad}^{-1}$.

Because we have significantly more measurements of $\alpha$ Tau than any other point-symmetric source, we use it as our primary closure phase calibrator. To determine the instrumental calibration for closure phase we have computed a weighted average of all of the $\alpha$ Tau measurements made from 2003 Aug through 2003 Nov, amounting to 16 nights of observation. This value of $(-26.51 \pm 0.32)^{\circ}$ is then removed from measurements made of other scientific targets. As evidenced by figure 2, our instrumental phase closure calibration remains reasonably constant over the five months of observations shown. Although the $\alpha$ Boo data is sparse, it appears to be consistent with the $\alpha$ Tau calibration; the instrumental phase offset has been subtracted from the data shown in figure 3.

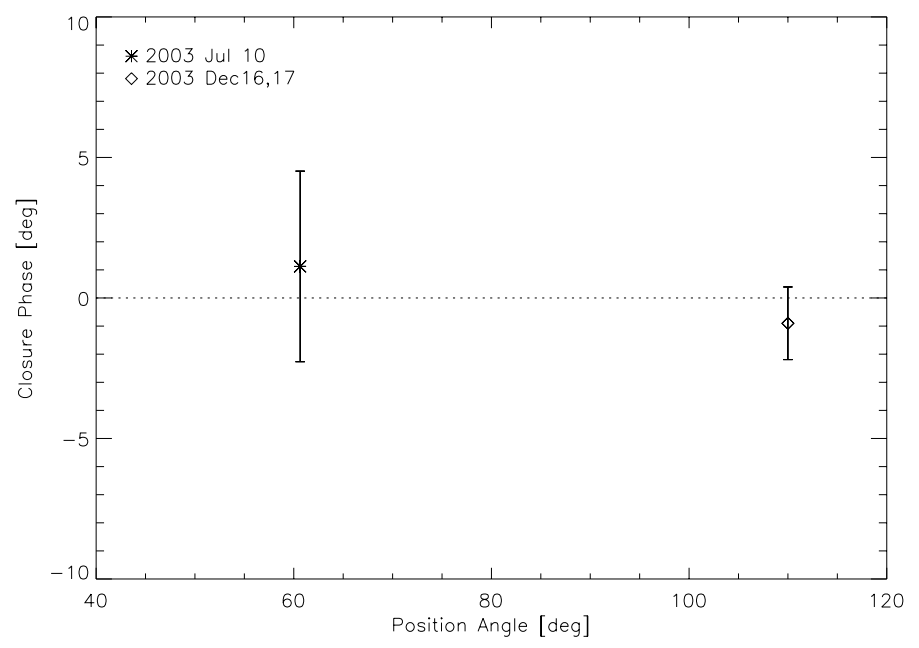

Figure 3. Closure phase data for $\alpha$ Boo. The instrumental calibration has been subtracted, and a line at $0^{\circ}$ is shown. The data span a range in spatial frequency $\sim(6-9) \times 10^{5} \mathrm{rad}^{-1}$.

Figure 4 shows the closure phase measurements for the M-type supergiant $\alpha$ Herculis. Although there appears to be a slight systematic departure from the $\alpha$ Tau calibration, it is small enough $(\lesssim 3 \sigma)$ to probably be negligible. The data are roughly consistent with a symmetric source over the $\sim 25^{\circ}$ of position angle covered. 
While earlier measurements by Danchi et al. ${ }^{8}$ indicated the presence of a dust shell around $\alpha$ Her in 1992 , more recent measurements by Close et al. ${ }^{9}$ in 2003 and the ISI in 2001* do not find any indication of dust at the spatial scales probed here. Although there may be some small amount of dust near the star, there is no strong evidence to suspect that $\alpha$ Her should be asymmetric at this resolution $\left(\sim 00^{\prime \prime} 09\right)$.

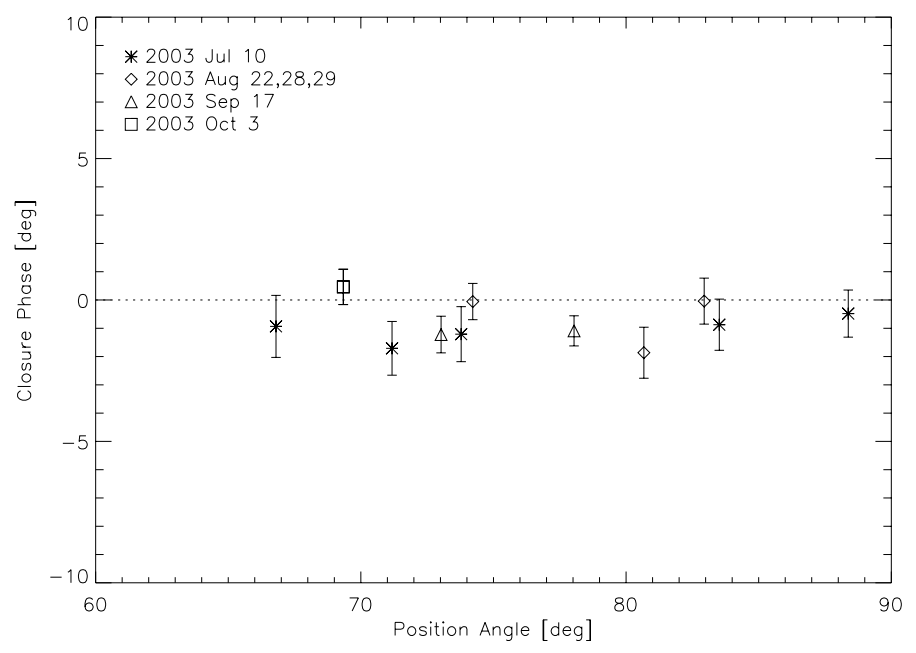

Figure 4. Calibrated closure phase data for $\alpha$ Her. The data span a range in spatial frequency $\sim(4-11) \times 10^{5} \mathrm{rad}^{-1}$.

Two examples of sources which do present clear evidence for asymmetries are the Mira-type variables NML Tauri and R Aquarii, whose closure phase data are presented in figure 5 and figure 6 (the latter will be discussed further in section 5.2.1). In the case of NML Tau, there is a marked change in the closure phase over the range of observations. Since both the position angle and the spatial frequency are changing, it is not clear whether the asymmetry is being resolved or rotated to an angle at which it is symmetric. At these spatial scales $(\sim 0$.' 1$)$, this may indicate the presence of one or more asymmetric dust shell "features"; further analysis of NML Tau will be given in a separate report.

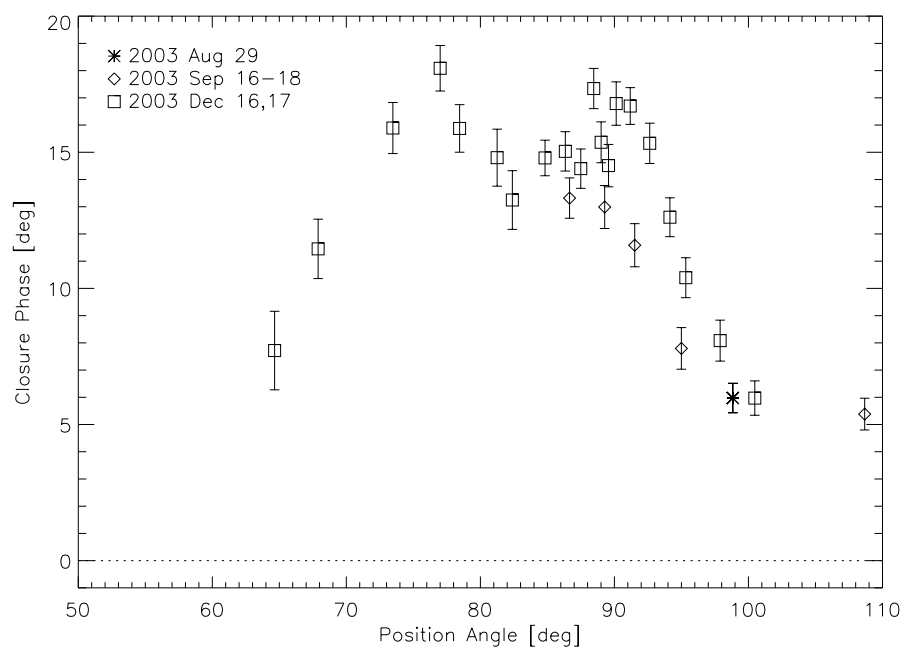

Figure 5. Calibrated closure phase data for NML Tau. The data span a range in spatial frequency $\sim(4-11) \times 10^{5} \mathrm{rad}^{-1}$.

\footnotetext{
${ }^{*}$ unpublished
} 
The closure phase measurements alone, though somewhat instructive, are not very useful by themselves, and we have also measured the visibilities for each of these sources. Using the measured visibility and closure phase information we can reconstruct the complex visibility (for the angles observed) and form a probable image for the star. This will be discussed in more detail in section 5. These examples of phase closure measurements have been given primarily to demonstrate the new capabilities of the ISI Array.

\section{IMAGE RECONSTRUCTION}

The complex phase of the visibility on any single baseline cannot be reconstructed from a single closure phase measured around a baseline triangle; the sum of the three phases does not include enough information to know any member of the sum directly. However, in some particular cases images can be constructed.

\subsection{Visibility Phase Calculation from Closure Phase}

Consider, for example, the case of three co-linear telescopes separated by baselines having the ratio $1: 2: 3$ (as is the current layout of the ISI), and assume that a star passes across the sky approximately in a single plane with the three telescopes. Then, the visibility magnitude and closure phase can be measured on a continuum of baselines ranging from 0 (assuming the star can be observed down to the horizon) to the longest baseline available. If $B$ is the shortest baseline, $\theta$ is the angle between the star and the zenith, and $x=\cos (\theta)$ is the projection, then the following measurements,

$$
\begin{gathered}
|\mathcal{V}(x)| \text { for } 0 \leq x \leq 3 B, \quad \text { and } \\
\Delta \Phi(x)=\phi(x)+\phi(2 x)+\phi(-3 x) \text { for } 0 \leq x \leq B
\end{gathered}
$$

are made, where $|\mathcal{V}(x)|$ is the visibility magnitude, $\Delta \Phi(x)$ is the closure phase, and $\phi(x)$ is the visibility phase. Since the image of the star on the sky is real, we have $\phi(x)=-\phi(-x)$ and so

$$
\Delta \Phi(x)=\phi(x)+\phi(2 x)-\phi(3 x) .
$$

The visibility phase can be reconstructed from the closure phase measurements by

$$
\phi(x)=-\sum_{m=0}^{\infty} \sum_{n=0}^{m}\left(\begin{array}{c}
m \\
n
\end{array}\right) \Delta \Phi\left(\frac{2^{n}}{3^{m+1}} x\right) .
$$

Therefore, if the measurements (3a) and (3b) are made, then the full complex visibility can be reconstructed for baselines up to $3 B$.

\subsection{ISI Imaging}

In practice, a lack of full spatial frequency coverage makes the approach of section 5.1 impractical. However, by extending a set of data in the most straight-forward manner possible, one can use this technique to construct the simplest image which is compatible with a limited set of data.

The baseline layout for initial testing purposes was chosen to be a closely separated $(4 \mathrm{~m}, 8 \mathrm{~m}, 12 \mathrm{~m})$ co-linear East-West array. Not only would this provide relatively strong fringe signals, but it would also allow for us to gain experience with the new data and utilize a fairly simple approach to image reconstructions. Although a co-linear array of telescopes will only provide information along a single projection, it does not have the position angle ambiguity that an arbitrary triangle of baselines would have. We can readily distinguish asymmetries at known position angles.

While it is theoretically possible to reconstruct the complex visibility from closure phase measurements, the ISI is not capable of measuring spatial frequencies all the way to zero. As a star nears the horizon, the atmospheric seeing becomes extremely poor, and other obstacles (such as trees or nearby buildings) may impede accurate measurements. Experience has shown that the ISI can obtain good visibility measurements down to an elevation of $\gtrsim 20^{\circ}$, providing for spatial frequencies of about one half of the maximum possible based upon the telescope separation. Because a complete continuum of measurements cannot be obtained, the data has to be extrapolated in order to reconstruct an image; a practical example of this is given in the following section. 


\subsubsection{R Aquarii}

In the case of the Mira-type variable star $\mathrm{R}$ Aquarii, a fairly complete visibility curve at resolutions typical of the circumstellar dust shell was recorded over two nights in 2003 Sep and Oct. The visibilities on all three baselines and the closure phases are plotted in figure 6 .
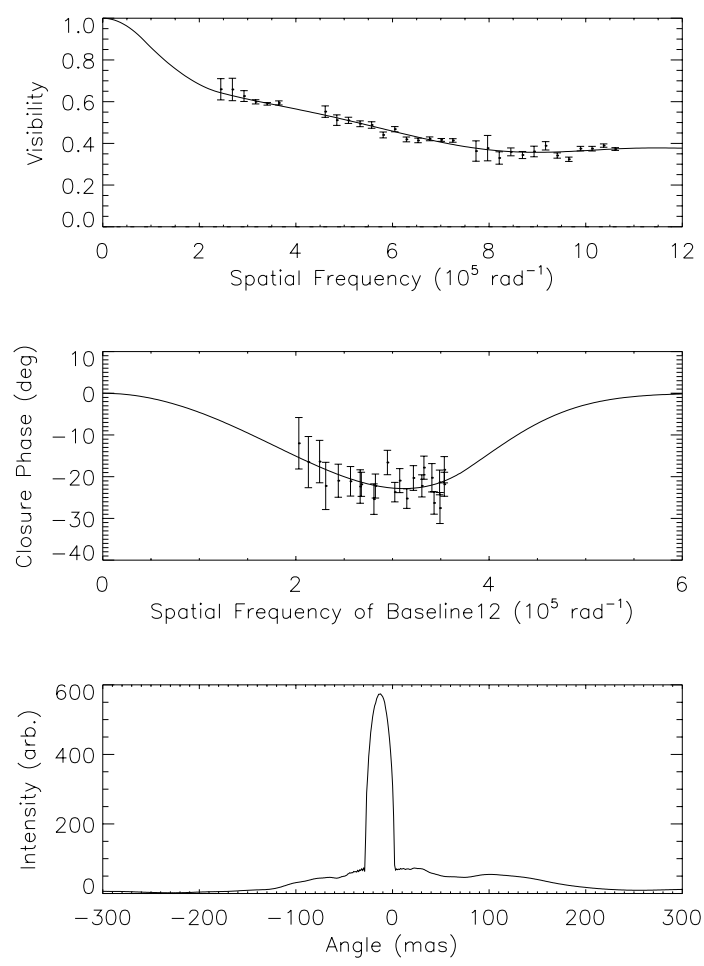

Figure 6. Visibility and closure phase data for R Aqr (top \& middle), with simple image reconstruction (bottom panel). The curves in the top \& middle panels are extrapolations beyond the measured data points.

For this example, at low resolutions the visibility was extrapolated to unity in a smooth fashion, and the closure phase was extrapolated to zero (containing only cubic terms and higher as must be the case). At high resolution, both the visibility and closure phase were extrapolated to coincide with those of a uniform symmetric disk 30 mas in diameter which contributes $35 \%$ of the total flux. The result of this simple extrapolation is a one-dimensional image, shown in the bottom panel of figure 6 . Although the actual stellar image is undoubtedly more complicated than this reconstruction, its general form, including the massive asymmetry in the dust shell, can be trusted.

\section{SUMMARY}

The simulations we presented at the SPIE Astronomical Telescopes \& Instrumentation Symposium held in Hawaii in $2002^{1}$ predicted that the measured error in phase closure for a three element heterodyne interferometer should be a few degrees or less for typically good fringe signals. The U.C. Berkeley ISI Array is now fully operational as an imaging interferometer array using heterodyne detection at mid-infrared (9-12 $\mu \mathrm{m})$ wavelengths, routinely measuring fringe visibilities on three simultaneous baselines, and one closure phase. Our actual measured closure phase error is typically less than a few degrees for two minute average, in good agreement with the earlier predictions, and for some of the more intense stars has been measured with a precision of less than one degree. Asymmetries have been measured for many stellar dust shells using a simple, co-linear 
E-W arrangement of baselines. We measure zero closure phases on astronomical point sources, and non-zero closure phases on several other stars suspected to be asymmetric. After some additional measurements have been made in the current configuration (anticipated mid- to late-2004), we expect to reorient the array into a

Y-configuration with perpendicular baselines on the order of $\sim 50 \mathrm{~m}$, where stellar disks can then be resolved.

\section{ACKNOWLEDGMENTS}

This work was supported by grants from the National Science Foundation, the Office of Naval Research, and the Gordon \& Betty Moore Foundation.

\section{REFERENCES}

1. D. D. S. Hale, W. Fitelson, J. D. Monnier, J. Weiner, and C. H. Townes, "Techniques for Measuring Phase Closure at 11 Microns," in Interferometry for Optical Astronomy II, Proc. SPIE 4838, 2003.

2. D. D. S. Hale, M. Bester, W. C. Danchi, W. Fitelson, S. Hoss, E. A. Lipman, J. D. Monnier, P. G. Tuthill, and C. H. Townes, "The Berkeley Infrared Spatial Interferometer: A heterodyne stellar interferometer for the mid-infrared," Astrophysical Journal 537, pp. 998-1012, 2000.

3. J. Weiner, D. D. S. Hale, and C. H. Townes, "Variability in late-type stellar diameters measured using mid-infrared interferometry," in Interferometry for Optical Astronomy II, Proc. SPIE 4838, 2003.

4. J. Weiner, D. D. S. Hale, and C. H. Townes, "Interferometry on Mira in the mid-infrared: Cyclic variability of the continuum diameter and the effect of spectral lines on apparent size," Astrophysical Journal 588, pp. 1064-1071, 2003.

5. J. Weiner, D. D. S. Hale, and C. H. Townes, "Asymptotic giant branch and supergiant stellar diameters in the mid-infrared," Astrophysical Journal 589, pp. 976-982, 2003.

6. D. D. S. Hale, "A Thermal Infrared Heterodyne Receiver with Applications to Astronomy," in Proc. NASA International Workshop on Thermal Detectors, B. Lakew, ed., TDW03, pp. 7-16 to 7-19, 2003.

7. C. Freed, "Design and Short-Term Stability of Single-Frequency $\mathrm{CO}_{2}$ Lasers," IEEE Journal of Quantum Electronics QE-4, no.6, p. 404, 1968.

8. W. C. Danchi, M. Bester, C. G. Degiacomi, L. J. Greenhill, and C. H. Townes, "Characteristics of Dust Shells around 13 Late-Type Stars," Astrophysical Journal 107, pp. 1469-1513, 1994.

9. L. M. Close, B. Biller, W. F. Hoffmann, P. M. Hinz, J. H. Bieging, F. Wildi, M. Lloyd-Hart, G. Brusa, D. Fisher, D. Miller, and R. Angel, "Mid-Infrared Imaging of the Post-Asymptotic Giant Branch Star AC Herculis with the Multiple Mirror Telescope Adaptive Optics System," Astrophysical Journal 598, pp. L35L38, 2003. 